Article

\title{
Well Posedness of New Optimization Problems with Variational Inequality Constraints
}

\author{
Savin Treanţă
}

check for updates

Citation: Treanţă, S. Well Posedness of New Optimization Problems with Variational Inequality Constraints. Fractal Fract. 2021, 5, 123. https:// doi.org/10.3390/ fractalfract5030123

Academic Editor: Hari Mohan Srivastava

Received: 18 August 2021

Accepted: 14 September 2021

Published: 15 September 2021

Publisher's Note: MDPI stays neutral with regard to jurisdictional claims in published maps and institutional affiliations.

Copyright: (c) 2021 by the authors. Licensee MDPI, Basel, Switzerland. This article is an open access article distributed under the terms and conditions of the Creative Commons Attribution (CC BY) license (https:// creativecommons.org/licenses/by/ $4.0 /)$.
Department of Applied Mathematics, University Politehnica of Bucharest, 060042 Bucharest, Romania; savin.treanta@upb.ro

\begin{abstract}
In this paper, we studied the well posedness for a new class of optimization problems with variational inequality constraints involving second-order partial derivatives. More precisely, by using the notions of lower semicontinuity, pseudomonotonicity, hemicontinuity and monotonicity for a multiple integral functional, and by introducing the set of approximating solutions for the considered class of constrained optimization problems, we established some characterization results on well posedness. Furthermore, to illustrate the theoretical developments included in this paper, we present some examples.
\end{abstract}

Keywords: well posedness; constrained variational control problem; monotonicity; pseudomonotonicity; hemicontinuity; multiple integral functional; lower semicontinuity

\section{Introduction}

The notion of well posedness represents a useful mathematical tool by ensuring the convergence of a sequence of approximate solutions to the exact solution of some optimization problems. Starting with the work of Tykhonov [1] for unconstrained optimization problems, various types of well posedness for variational problems have been considered (see, for instance, Levitin-Polyak well posedness [2-5], extended well posedness [6-14]), $L$-well posedness [15], $\alpha$-well posedness [16,17]). Moreover, the concept of well posedness can be useful to study some related problems, such as variational inequality and fixed point problems [18-22], hemivariational inequality problems [23], complementary problems [24], equilibrium problems [25,26], Nash equilibrium problems [27] and variational inclusion problems [28]. Recently, the study of well posedness for vector variational inequalities and the associated optimization problems was formulated by Jayswal and Shalini [29]. On the other hand, an important and interesting extension of variational inequality problems is that of multidimensional variational inequality problems and the corresponding multi-time optimization problems (see [30-40]).

Motivated by the aforementioned research works, in this paper we analyze the well posedness of a new class of constrained optimization problems governed by multiple integral functionals involving second-order partial derivatives. To this aim, first we introduce new forms for the concepts of monotonicity, lower semicontinuity, pseudomonotonicity and hemicontinuity associated with a multiple integral functional. Furthermore, we define the set of approximating solutions for the considered optimization problem and establish some characterization theorems on well posedness. The main novelty elements of this paper are represented by the following: the mathematical framework is based on infinitedimensional function spaces, multiple integral functionals, the presence of second-order partial derivatives, and innovative proofs of the main results. The aforementioned elements are completely new in the area of well-posed variational control problems. Most of the previous works in this field have been studied in classical finite-dimensional spaces, without taking into account the new notions mentioned above.

This paper is organized as follows. Section 2 provides the concepts of monotonicity, pseudomonotonicity, hemicontinuity and the lower semicontinuity of a multiple integral 
functional, and an auxiliary lemma. Section 3 investigates the well posedness for the considered constrained optimization problem. Concretely, we establish that well posedness and the existence and uniqueness of a solution are equivalent in the aforementioned problem. Furthermore, some examples are formulated throughout the paper to highlight the theoretical elements. Finally, in Section 4, we present the conclusions and provide further developments.

\section{Preliminaries}

Throughout this work, we consider the following mathematical tools and notations: let $\Omega$ be a compact set in $\mathbb{R}^{m}$ and $\Omega \ni \zeta=\left(\zeta^{\alpha}\right), \alpha=\overline{1, m}$; consider $\mathcal{A}$ as the space of $C^{4}$-class state functions $a: \Omega \rightarrow \mathbb{R}^{n}$ and $a_{\alpha}:=\frac{\partial a}{\partial \zeta^{\alpha}}, a_{\beta \gamma}:=\frac{\partial^{2} a}{\partial \zeta^{\beta} \partial \zeta^{\gamma}}$ denote the partial speed and partial acceleration, respectively; also, consider $\mathcal{U}$ ass the space of $C^{1}$-class control functions $u: \Omega \rightarrow \mathbb{R}^{k}$, and consider $A \times U$ as a closed, convex and non-empty subset of $\mathcal{A} \times \mathcal{U},\left.(a, u)\right|_{\partial \Omega}=$ given, endowed with the scalar product:

$$
\begin{gathered}
\langle(a, u),(b, w)\rangle=\int_{\Omega}[a(\zeta) \cdot b(\zeta)+u(\zeta) \cdot w(\zeta)] d \zeta \\
=\int_{\Omega}\left[\sum_{i=1}^{n} a^{i}(\zeta) b^{i}(\zeta)+\sum_{j=1}^{k} u^{j}(\zeta) w^{j}(\zeta)\right] d \zeta, \quad \forall(a, u),(b, w) \in \mathcal{A} \times \mathcal{U}
\end{gathered}
$$

and the induced norm, where $d \zeta=d \zeta^{1} \cdots d \zeta^{m}$ denotes the volume element on $\mathbb{R}^{m}$.

Consider $J^{2}\left(\mathbb{R}^{m}, \mathbb{R}^{n}\right)$ as the second-order jet bundle associated with $\mathbb{R}^{m}$ and $\mathbb{R}^{n}$. Taking the scalar continuously differentiable function $f: J^{2}\left(\mathbb{R}^{m}, \mathbb{R}^{n}\right) \times \mathbb{R}^{k} \rightarrow \mathbb{R}$, we introduce the following multiple integral-type functional:

$$
F: \mathcal{A} \times \mathcal{U} \rightarrow \mathbb{R}, \quad F(a, u)=\int_{\Omega} f\left(\zeta, a(\zeta), a_{\alpha}(\zeta), a_{\beta \gamma}(\zeta), u(\zeta)\right) d \zeta .
$$

At this moment, we are able to introduce the following constrained variational control problem (in short, CVCP), given as follows (we use the notation $\left(\pi_{a, u}(\zeta)\right):=\left(\zeta, a(\zeta), a_{\alpha}(\zeta)\right.$, $\left.\left.a_{\beta \gamma}(\zeta), u(\zeta)\right)\right)$ :

$$
\begin{array}{ll}
\text { (CVCP) } & \text { Minimize }_{(a, u)} \int_{\Omega} f\left(\pi_{a, u}(\zeta)\right) d \zeta \\
& \text { subject to }(a, u) \in \Theta
\end{array}
$$

where $\Theta$ is the solution set of the controlled variational inequality problem (in short, CVIP): to find a pair $(a, u) \in A \times U$ such that:

$$
\begin{aligned}
(\mathrm{CVIP}) \quad \int_{\Omega} & {\left[\frac{\partial f}{\partial a}\left(\pi_{a, u}(\zeta)\right)(b(\zeta)-a(\zeta))+\frac{\partial f}{\partial a_{\alpha}}\left(\pi_{a, u}(\zeta)\right) D_{\alpha}(b(\zeta)-a(\zeta))\right.} \\
& +\frac{1}{n(\beta, \gamma)} \frac{\partial f}{\partial a_{\beta \gamma}}\left(\pi_{a, u}(\zeta)\right) D_{\beta \gamma}^{2}(b(\zeta)-a(\zeta)) \\
+ & \left.\frac{\partial f}{\partial u}\left(\pi_{a, u}(\zeta)\right)(w(\zeta)-u(\zeta))\right] d \zeta \geq 0, \quad \forall(b, w) \in A \times U
\end{aligned}
$$

where $D_{\beta \gamma}^{2}:=D_{\beta}\left(D_{\gamma}\right)$, and $n(\beta, \gamma)$ is the Saunders's multi-index notation (see Saunders [41], Treanţă [40]). 
More precisely, the feasible solution set for (CVIP) is given by

$$
\begin{gathered}
\Theta=\left\{(a, u) \in A \times U: \int_{\Omega}\left[(b(\zeta)-a(\zeta)) \frac{\partial f}{\partial a}\left(\pi_{a, u}(\zeta)\right)\right.\right. \\
+D_{\alpha}(b(\zeta)-a(\zeta)) \frac{\partial f}{\partial a_{\alpha}}\left(\pi_{a, u}(\zeta)\right)+(w(\zeta)-u(\zeta)) \frac{\partial f}{\partial u}\left(\pi_{a, u}(\zeta)\right) \\
\left.+\frac{1}{n(\beta, \gamma)} D_{\beta \gamma}^{2}(b(\zeta)-a(\zeta)) \frac{\partial f}{\partial a_{\beta \gamma}}\left(\pi_{a, u}(\zeta)\right)\right] d \zeta \geq 0 \\
\forall(b, w) \in A \times U\} .
\end{gathered}
$$

Next, we define the notions of monotonicity and pseudomonotonicity for the aforementioned multiple integral functional.

Definition 1. The multiple integral functional $F(a, u)=\int_{\Omega} f\left(\pi_{a, u}(\zeta)\right) d \zeta$ is called monotone on $A \times U$ if the following inequality holds:

$$
\begin{aligned}
& \int_{\Omega}\left[(a(\zeta)-b(\zeta))\left(\frac{\partial f}{\partial a}\left(\pi_{a, u}(\zeta)\right)-\frac{\partial f}{\partial a}\left(\pi_{b, w}(\zeta)\right)\right)\right. \\
& +(u(\zeta)-w(\zeta))\left(\frac{\partial f}{\partial u}\left(\pi_{a, u}(\zeta)\right)-\frac{\partial f}{\partial u}\left(\pi_{b, w}(\zeta)\right)\right) \\
& +D_{\alpha}(a(\zeta)-b(\zeta))\left(\frac{\partial f}{\partial a_{\alpha}}\left(\pi_{a, u}(\zeta)\right)-\frac{\partial f}{\partial a_{\alpha}}\left(\pi_{b, w}(\zeta)\right)\right) \\
& \left.+\frac{1}{n(\beta, \gamma)} D_{\beta \gamma}^{2}(a(\zeta)-b(\zeta))\left(\frac{\partial f}{\partial a_{\beta \gamma}}\left(\pi_{a, u}(\zeta)\right)-\frac{\partial f}{\partial a_{\beta \gamma}}\left(\pi_{b, w}(\zeta)\right)\right)\right] d \zeta \geq 0, \\
& \forall(a, u),(b, w) \in A \times U .
\end{aligned}
$$

Definition 2. The multiple integral functional $F(a, u)=\int_{\Omega} f\left(\pi_{a, u}(\zeta)\right) d \zeta$ is called pseudomonotone on $A \times U$ if the following implication holds:

$$
\begin{gathered}
\int_{\Omega}\left[(a(\zeta)-b(\zeta)) \frac{\partial f}{\partial a}\left(\pi_{b, w}(\zeta)\right)+(u(\zeta)-w(\zeta)) \frac{\partial f}{\partial u}\left(\pi_{b, w}(\zeta)\right)\right. \\
+D_{\alpha}(a(\zeta)-b(\zeta)) \frac{\partial f}{\partial a_{\alpha}}\left(\pi_{b, w}(\zeta)\right) \\
\left.+\frac{1}{n(\beta, \gamma)} D_{\beta \gamma}^{2}(a(\zeta)-b(\zeta)) \frac{\partial f}{\partial a_{\beta \gamma}}\left(\pi_{b, w}(\zeta)\right)\right] d \zeta \geq 0 \\
\Rightarrow \quad \int_{\Omega}\left[(a(\zeta)-b(\zeta)) \frac{\partial f}{\partial a}\left(\pi_{a, u}(\zeta)\right)+(u(\zeta)-w(\zeta)) \frac{\partial f}{\partial u}\left(\pi_{a, u}(\zeta)\right)\right. \\
+D_{\alpha}(a(\zeta)-b(\zeta)) \frac{\partial f}{\partial a_{\alpha}}\left(\pi_{a, u}(\zeta)\right) \\
\left.+\frac{1}{n(\beta, \gamma)} D_{\beta \gamma}^{2}(a(\zeta)-b(\zeta)) \frac{\partial f}{\partial a_{\beta \gamma}}\left(\pi_{a, u}(\zeta)\right)\right] d \zeta \geq 0, \quad \forall(a, u),(b, w) \in A \times U
\end{gathered}
$$

Let us give an example of a multiple integral-type functional which is not monotone but is pseudomonotone.

Example 1. Consider $n=k=1, m=2$, and $\Omega=[0,3]^{2}$. We define:

$$
f\left(\pi_{a, u}(\zeta)\right)=2 \sin a(\zeta)+u(\zeta) e^{u(\zeta)}
$$


and show, in accordance with Definition 2, that the multiple integral functional $F(a, u)=$ $\int_{\Omega} f\left(\pi_{a, u}(\zeta)\right) d \zeta$ is pseudomonotone on $A \times U=C^{4}(\Omega,[-1,1]) \times C^{1}(\Omega,[-1,1])$. Indeed, we have:

$$
\begin{gathered}
\int_{\Omega}\left[(a(\zeta)-b(\zeta)) \frac{\partial f}{\partial a}\left(\pi_{b, w}(\zeta)\right)+(u(\zeta)-w(\zeta)) \frac{\partial f}{\partial u}\left(\pi_{b, w}(\zeta)\right)\right. \\
+D_{\alpha}(a(\zeta)-b(\zeta)) \frac{\partial f}{\partial a_{\alpha}}\left(\pi_{b, w}(\zeta)\right) \\
\left.+\frac{1}{n(\beta, \gamma)} D_{\beta \gamma}^{2}(a(\zeta)-b(\zeta)) \frac{\partial f}{\partial a_{\beta \gamma}}\left(\pi_{b, w}(\zeta)\right)\right] d \zeta \\
=\int_{\Omega}\left[2(a(\zeta)-b(\zeta)) \cos b(\zeta)+(u(\zeta)-w(\zeta))\left(e^{w(\zeta)}+w(\zeta) e^{w(\zeta)}\right)\right] d \zeta \geq 0, \\
\forall(a, u),(b, w) \in A \times U \\
\Rightarrow \int_{\Omega}\left[(a(\zeta)-b(\zeta)) \frac{\partial f}{\partial a}\left(\pi_{a, u}(\zeta)\right)+(u(\zeta)-w(\zeta)) \frac{\partial f}{\partial u}\left(\pi_{a, u}(\zeta)\right)\right. \\
+D_{\alpha}(a(\zeta)-b(\zeta)) \frac{\partial f}{\partial a_{\alpha}}\left(\pi_{a, u}(\zeta)\right) \\
\left.+\frac{1}{n(\beta, \gamma)} D_{\beta \gamma}^{2}(a(\zeta)-b(\zeta)) \frac{\partial f}{\partial a_{\beta \gamma}}\left(\pi_{a, u}(\zeta)\right)\right] d \zeta \\
=\int_{\Omega}\left[2(a(\zeta)-b(\zeta)) \cos a(\zeta)+(u(\zeta)-w(\zeta))\left(e^{u(\zeta)}+u(\zeta) e^{u(\zeta)}\right)\right] d \zeta \geq 0, \\
\forall(a, u),(b, w) \in A \times U .
\end{gathered}
$$

However, it is not monotone on $A \times U$ in the sense of Definition 1, because:

$$
\begin{gathered}
\int_{\Omega}\left[(a(\zeta)-b(\zeta))\left(\frac{\partial f}{\partial a}\left(\pi_{a, u}(\zeta)\right)-\frac{\partial f}{\partial a}\left(\pi_{b, w}(\zeta)\right)\right)\right. \\
+(u(\zeta)-w(\zeta))\left(\frac{\partial f}{\partial u}\left(\pi_{a, u}(\zeta)\right)-\frac{\partial f}{\partial u}\left(\pi_{b, w}(\zeta)\right)\right) \\
+D_{\alpha}(a(\zeta)-b(\zeta))\left(\frac{\partial f}{\partial a_{\alpha}}\left(\pi_{a, u}(\zeta)\right)-\frac{\partial f}{\partial a_{\alpha}}\left(\pi_{b, w}(\zeta)\right)\right) \\
\left.+\frac{1}{n(\beta, \gamma)} D_{\beta \gamma}^{2}(a(\zeta)-b(\zeta))\left(\frac{\partial f}{\partial a_{\beta \gamma}}\left(\pi_{a, u}(\zeta)\right)-\frac{\partial f}{\partial a_{\beta \gamma}}\left(\pi_{b, w}(\zeta)\right)\right)\right] d \zeta \\
=\int_{\Omega}[2(a(\zeta)-b(\zeta))(\cos a(\zeta)-\cos b(\zeta)) \\
\left.+(u(\zeta)-w(\zeta))\left(u(\zeta) e^{u(\zeta)}+e^{u(\zeta)}-w(\zeta) e^{w(\zeta)}-e^{w(\zeta)}\right)\right] d \zeta \ngtr 0, \\
\forall(a, u),(b, w) \in A \times U .
\end{gathered}
$$

Then, in accordance with Usman and Khan [42], we define the concept of hemicontinuity for the considered multiple integral-type functional.

Definition 3. The functional $F(a, u)=\int_{\Omega} f\left(\pi_{a, u}(\zeta)\right) d \zeta$ is hemicontinuous on $A \times U$ if the application:

$$
\lambda \rightarrow\left\langle\left((a(\zeta), u(\zeta))-(b(\zeta), w(\zeta)),\left(\frac{\delta F}{\delta a_{\lambda}}(\zeta), \frac{\delta F}{\delta u_{\lambda}}(\zeta)\right)\right\rangle, \quad 0 \leq \lambda \leq 1\right.
$$


is continuous at $0^{+}$, for $\forall(a, u),(b, w) \in A \times U$, where:

$$
\begin{gathered}
\frac{\delta F}{\delta a_{\lambda}}(\zeta):=\frac{\partial f}{\partial a}\left(\pi_{a_{\lambda}, u_{\lambda}}(\zeta)\right)-D_{\alpha} \frac{\partial f}{\partial a_{\alpha}}\left(\pi_{a_{\lambda}, u_{\lambda}}(\zeta)\right)+\frac{1}{n(\beta, \gamma)} D_{\beta \gamma}^{2} \frac{\partial f}{\partial a_{\beta \gamma}}\left(\pi_{a_{\lambda}, u_{\lambda}}(\zeta)\right) \in A, \\
\frac{\delta F}{\delta u_{\lambda}}(\zeta):=\frac{\partial f}{\partial u}\left(\pi_{a_{\lambda}, u_{\lambda}}(\zeta)\right) \in U, \\
a_{\lambda}:=\lambda a+(1-\lambda) b, \quad u_{\lambda}:=\lambda u+(1-\lambda) w .
\end{gathered}
$$

The following lemma is an auxiliary result for proving the main results derived in the present paper.

Lemma 1. Consider $F(a, u)=\int_{\Omega} f\left(\pi_{a, u}(\zeta)\right) d \zeta$ is pseudomonotone and hemicontinuous on $A \times U$. The pair $(a, u) \in A \times U$ is a solution for (CVIP) if and only if $(a, u)$ is a solution for the following variational inequality problem:

$$
\begin{gathered}
\int_{\Omega}\left[(b(\zeta)-a(\zeta)) \frac{\partial f}{\partial a}\left(\pi_{b, w}(\zeta)\right)+(w(\zeta)-u(\zeta)) \frac{\partial f}{\partial u}\left(\pi_{b, w}(\zeta)\right)\right. \\
+D_{\alpha}(b(\zeta)-a(\zeta)) \frac{\partial f}{\partial a_{\alpha}}\left(\pi_{b, w}(\zeta)\right) \\
\left.+\frac{1}{n(\beta, \gamma)} D_{\beta \gamma}^{2}(b(\zeta)-a(\zeta)) \frac{\partial f}{\partial a_{\beta \gamma}}\left(\pi_{b, w}(\zeta)\right)\right] d \zeta \geq 0, \quad \forall(b, w) \in A \times U .
\end{gathered}
$$

Proof. Consider that the pair $(a, u) \in A \times U$ is the solution for (CVIP). As a consequence, it results that:

$$
\begin{gathered}
\int_{\Omega}\left[(b(\zeta)-a(\zeta)) \frac{\partial f}{\partial a}\left(\pi_{a, u}(\zeta)\right)+(w(\zeta)-u(\zeta)) \frac{\partial f}{\partial u}\left(\pi_{a, u}(\zeta)\right)\right. \\
+D_{\alpha}(b(\zeta)-a(\zeta)) \frac{\partial f}{\partial a_{\alpha}}\left(\pi_{a, u}(\zeta)\right) \\
\left.+\frac{1}{n(\beta, \gamma)} D_{\beta \gamma}^{2}(b(\zeta)-a(\zeta)) \frac{\partial f}{\partial a_{\beta \gamma}}\left(\pi_{a, u}(\zeta)\right)\right] d \zeta \geq 0, \quad \forall(b, w) \in A \times U .
\end{gathered}
$$

By using the pseudomonotonicity property of the considered multiple integral functional (see Definition 2), the previous inequality involves:

$$
\begin{gathered}
\int_{\Omega}\left[(b(\zeta)-a(\zeta)) \frac{\partial f}{\partial a}\left(\pi_{b, w}(\zeta)\right)+(w(\zeta)-u(\zeta)) \frac{\partial f}{\partial u}\left(\pi_{b, w}(\zeta)\right)\right. \\
+D_{\alpha}(b(\zeta)-a(\zeta)) \frac{\partial f}{\partial a_{\alpha}}\left(\pi_{b, w}(\zeta)\right) \\
\left.+\frac{1}{n(\beta, \gamma)} D_{\beta \gamma}^{2}(b(\zeta)-a(\zeta)) \frac{\partial f}{\partial a_{\beta \gamma}}\left(\pi_{b, w}(\zeta)\right)\right] d \zeta \geq 0, \quad \forall(b, w) \in A \times U .
\end{gathered}
$$

Conversely, assume that:

$$
\begin{gathered}
\int_{\Omega}\left[(b(\zeta)-a(\zeta)) \frac{\partial f}{\partial a}\left(\pi_{b, w}(\zeta)\right)+(w(\zeta)-u(\zeta)) \frac{\partial f}{\partial u}\left(\pi_{b, w}(\zeta)\right)\right. \\
+D_{\alpha}(b(\zeta)-a(\zeta)) \frac{\partial f}{\partial a_{\alpha}}\left(\pi_{b, w}(\zeta)\right) \\
\left.+\frac{1}{n(\beta, \gamma)} D_{\beta \gamma}^{2}(b(\zeta)-a(\zeta)) \frac{\partial f}{\partial a_{\beta \gamma}}\left(\pi_{b, w}(\zeta)\right)\right] d \zeta \geq 0, \quad \forall(b, w) \in A \times U .
\end{gathered}
$$


For $\lambda \in(0,1]$ and $(b, w) \in A \times U$, we define:

$$
\left(b_{\lambda}, w_{\lambda}\right)=((1-\lambda) a+\lambda b,(1-\lambda) u+\lambda w) \in A \times U .
$$

Thus, the above inequality implies:

$$
\begin{gathered}
\int_{\Omega}\left[\left(b_{\lambda}(\zeta)-a(\zeta)\right) \frac{\partial f}{\partial a}\left(\pi_{b_{\lambda}, w_{\lambda}}(\zeta)\right)+\left(w_{\lambda}(\zeta)-u(\zeta)\right) \frac{\partial f}{\partial u}\left(\pi_{b_{\lambda}, w_{\lambda}}(\zeta)\right)\right. \\
+D_{\alpha}\left(b_{\lambda}(\zeta)-a(\zeta)\right) \frac{\partial f}{\partial a_{\alpha}}\left(\pi_{b_{\lambda}, w_{\lambda}}(\zeta)\right) \\
\left.+\frac{1}{n(\beta, \gamma)} D_{\beta \gamma}^{2}\left(b_{\lambda}(\zeta)-a(\zeta)\right) \frac{\partial f}{\partial a_{\beta \gamma}}\left(\pi_{b_{\lambda}, w_{\lambda}}(\zeta)\right)\right] d \zeta \geq 0, \quad(b, w) \in A \times U .
\end{gathered}
$$

By considering $\lambda \rightarrow 0$, we obtain:

$$
\begin{gathered}
\int_{\Omega}\left[(b(\zeta)-a(\zeta)) \frac{\partial f}{\partial a}\left(\pi_{a, u}(\zeta)\right)+(w(\zeta)-u(\zeta)) \frac{\partial f}{\partial u}\left(\pi_{a, u}(\zeta)\right)\right. \\
+D_{\alpha}(b(\zeta)-a(\zeta)) \frac{\partial f}{\partial a_{\alpha}}\left(\pi_{a, u}(\zeta)\right) \\
\left.+\frac{1}{n(\beta, \gamma)} D_{\beta \gamma}^{2}(b(\zeta)-a(\zeta)) \frac{\partial f}{\partial a_{\beta \gamma}}\left(\pi_{a, u}(\zeta)\right)\right] d \zeta \geq 0, \quad \forall(b, w) \in A \times U,
\end{gathered}
$$

which proves that $(a, u)$ solves (CVIP). This completes the proof of this lemma.

Now, we give the definition of lower semicontinuity for the multiple integral functional $F(a, u)=\int_{\Omega} f\left(\pi_{a, u}(\zeta)\right) d \zeta$.

Definition 4. The multiple integral functional $F(a, u)=\int_{\Omega} f\left(\pi_{a, u}(\zeta)\right) d \zeta$ is called lower semicontinuous at a point $\left(a_{0}, u_{0}\right) \in A \times U$ if:

$$
\int_{\Omega} f\left(\pi_{a_{0}, u_{0}}(\zeta)\right) d \zeta \leq \lim _{(a, u) \rightarrow\left(a_{0}, u_{0}\right)} \inf \int_{\Omega} f\left(\pi_{a, u}(\zeta)\right) d \zeta
$$

\section{Well Posedness Associated with (CVCP)}

In this section, by considering the notions introduced in Section 2, we study the well posedness for the considered class of constrained optimization problems (CVCPs). To this aim, we introduce the following definitions and notations.

Denote by $\mathcal{S}$ solution set of (CVCP), namely:

$$
\begin{aligned}
& \mathcal{S}=\left\{(a, u) \in A \times U \mid \int_{\Omega} f\left(\pi_{a, u}(\zeta)\right) d \zeta \leq \inf _{(b, w) \in \Theta} \int_{\Omega} f\left(\pi_{b, w}(\zeta)\right) d \zeta\right. \text { and } \\
& \int_{\Omega}\left[(b(\zeta)-a(\zeta)) \frac{\partial f}{\partial a}\left(\pi_{a, u}(\zeta)\right)+(w(\zeta)-u(\zeta)) \frac{\partial f}{\partial u}\left(\pi_{a, u}(\zeta)\right)\right. \\
& +D_{\alpha}(b(\zeta)-a(\zeta)) \frac{\partial f}{\partial a_{\alpha}}\left(\pi_{a, u}(\zeta)\right) \\
& \left.\left.+\frac{1}{n(\beta, \gamma)} D_{\beta \gamma}^{2}(b(\zeta)-a(\zeta)) \frac{\partial f}{\partial a_{\beta \gamma}}\left(\pi_{a, u}(\zeta)\right)\right] d \zeta \geq 0, \forall(b, w) \in A \times U\right\} .
\end{aligned}
$$


Consider the set of approximating solutions of (CVCP), for $\sigma, \iota \geq 0$, as follows:

$$
\begin{aligned}
& \mathcal{S}(\sigma, \iota)=\left\{(a, u) \in A \times U \mid \int_{\Omega} f\left(\pi_{a, u}(\zeta)\right) d \zeta \leq \inf _{(b, w) \in \Theta} \int_{\Omega} f\left(\pi_{b, w}(\zeta)\right) d \zeta+\sigma\right. \text { and } \\
& \int_{\Omega}\left[(b(\zeta)-a(\zeta)) \frac{\partial f}{\partial a}\left(\pi_{a, u}(\zeta)\right)+(w(\zeta)-u(\zeta)) \frac{\partial f}{\partial u}\left(\pi_{a, u}(\zeta)\right)\right. \\
& +D_{\alpha}(b(\zeta)-a(\zeta)) \frac{\partial f}{\partial a_{\alpha}}\left(\pi_{a, u}(\zeta)\right) \\
& \left.\left.+\frac{1}{n(\beta, \gamma)} D_{\beta \gamma}^{2}(b(\zeta)-a(\zeta)) \frac{\partial f}{\partial a_{\beta \gamma}}\left(\pi_{a, u}(\zeta)\right)\right] d \zeta+\iota \geq 0, \forall(b, w) \in A \times U\right\} .
\end{aligned}
$$

Remark 1. For $(\sigma, \iota)=(0,0)$, we obtain $\mathcal{S}=\mathcal{S}(\sigma, \iota)$, and for $(\sigma, \iota)>(0,0)$, we obtain $\mathcal{S} \subseteq \mathcal{S}(\sigma, \iota)$

Definition 5. The sequence $\left\{\left(a_{n}, u_{n}\right)\right\}$ is an approximating sequence for (CVCP) if there exists $\iota_{n} \rightarrow 0$ (a sequence of positive real numbers) as $n \rightarrow \infty$, such that:

$$
\lim _{n \rightarrow \infty} \sup \int_{\Omega} f\left(\pi_{a_{n}, u_{n}}(\zeta)\right) d \zeta \leq \inf _{(b, w) \in \Theta} \int_{\Omega} f\left(\pi_{b, w}(\zeta)\right) d \zeta
$$

and:

$$
\begin{gathered}
\int_{\Omega}\left[\left(b(\zeta)-a_{n}(\zeta)\right) \frac{\partial f}{\partial a}\left(\pi_{a_{n}, u_{n}}(\zeta)\right)+\left(w(\zeta)-u_{n}(\zeta)\right) \frac{\partial f}{\partial u}\left(\pi_{a_{n}, u_{n}}(\zeta)\right)\right. \\
+D_{\alpha}\left(b(\zeta)-a_{n}(\zeta)\right) \frac{\partial f}{\partial a_{\alpha}}\left(\pi_{a_{n}, u_{n}}(\zeta)\right) \\
\left.+\frac{1}{n(\beta, \gamma)} D_{\beta \gamma}^{2}\left(b(\zeta)-a_{n}(\zeta)\right) \frac{\partial f}{\partial a_{\beta \gamma}}\left(\pi_{a_{n}, u_{n}}(\zeta)\right)\right] d \zeta+\iota_{n} \geq 0, \quad \forall(b, w) \in A \times U
\end{gathered}
$$

are fulfilled.

Definition 6. The constrained optimization (CVCP) is well posed if:

(i) it admits a single solution $\left(a_{0}, u_{0}\right)$;

and (ii) each approximating sequence of (CVCP) converges to $\left(a_{0}, u_{0}\right)$.

Furthermore, denote by "diam $B$ " the diameter of the set $B$ and it is defined as follows

$$
\operatorname{diam} B=\sup _{x, y \in B}\|x-y\| .
$$

The next theorem represents a first characterization result on the well posedness for (CVCP).

Theorem 1. Consider that $F(a, u)=\int_{\Omega} f\left(\pi_{a, u}(\zeta)\right) d \zeta$ is lower semicontinuous, monotone and hemicontinuous on $A \times U$. The constrained optimization problem (CVCP) is well posed if and only if:

$$
\mathcal{S}(\sigma, \iota) \neq \varnothing, \forall \sigma, \iota>0 \text { and diam } \mathcal{S}(\sigma, \iota) \rightarrow 0 \text { as }(\sigma, \iota) \rightarrow(0,0) .
$$

Proof. Consider (CVCP) is well posed. In consequence, it admits a single solution $(\bar{a}, \bar{u}) \in$ $\mathcal{S}$. By using the inclusion $\mathcal{S} \subseteq \mathcal{S}(\sigma, \iota), \forall \sigma, \iota>0$, we obtain $\mathcal{S}(\sigma, \iota) \neq \varnothing, \forall \sigma, \iota>0$. Now, contrary to the result, suppose that diam $\mathcal{S}(\sigma, l) \nrightarrow 0$ as $(\sigma, l) \rightarrow(0,0)$. Consequently, there exists $r>0$, a positive integer $m, \sigma_{n}, \iota_{n}>0$ with $\sigma_{n}, \iota_{n} \rightarrow 0$, and $\left(a_{n}, u_{n}\right),\left(a_{n}^{\prime}, u_{n}^{\prime}\right) \in$ $\mathcal{S}\left(\sigma_{n}, l_{n}\right)$ such that:

$$
\left\|\left(a_{n}, u_{n}\right)-\left(a_{n}^{\prime}, u_{n}^{\prime}\right)\right\|>r, \quad \forall n \geq m .
$$


Since $\left(a_{n}, u_{n}\right),\left(a_{n}^{\prime}, u_{n}^{\prime}\right) \in \mathcal{S}\left(\sigma_{n}, \iota_{n}\right)$, we obtain:

$$
\begin{gathered}
\int_{\Omega} f\left(\pi_{a_{n}, u_{n}}(\zeta)\right) d \zeta \leq \inf _{(b, w) \in \Theta} \int_{\Omega} f\left(\pi_{b, w}(\zeta)\right) d \zeta+\sigma_{n} \\
\int_{\Omega}\left[\left(b(\zeta)-a_{n}(\zeta)\right) \frac{\partial f}{\partial a}\left(\pi_{a_{n}, u_{n}}(\zeta)\right)+\left(w(\zeta)-u_{n}(\zeta)\right) \frac{\partial f}{\partial u}\left(\pi_{a_{n}, u_{n}}(\zeta)\right)\right. \\
+D_{\alpha}\left(b(\zeta)-a_{n}(\zeta)\right) \frac{\partial f}{\partial a_{\alpha}}\left(\pi_{a_{n}, u_{n}}(\zeta)\right) \\
\left.+\frac{1}{n(\beta, \gamma)} D_{\beta \gamma}^{2}\left(b(\zeta)-a_{n}(\zeta)\right) \frac{\partial f}{\partial a_{\beta \gamma}}\left(\pi_{a_{n}, u_{n}}(\zeta)\right)\right] d \zeta+\iota_{n} \geq 0, \quad \forall(b, w) \in A \times U
\end{gathered}
$$

and:

$$
\begin{gathered}
\int_{\Omega} f\left(\pi_{a_{n}^{\prime}, u_{n}^{\prime}}(\zeta)\right) d \zeta \leq \inf _{(b, w) \in \Theta} \int_{\Omega} f\left(\pi_{b, w}(\zeta)\right) d \zeta+\sigma_{n} \\
\int_{\Omega}\left[\left(b(\zeta)-a_{n}^{\prime}(\zeta)\right) \frac{\partial f}{\partial a}\left(\pi_{a_{n}^{\prime}, u_{n}^{\prime}}(\zeta)\right)+\left(w(\zeta)-u_{n}^{\prime}(\zeta)\right) \frac{\partial f}{\partial u}\left(\pi_{a_{n}^{\prime}, u_{n}^{\prime}}(\zeta)\right)\right. \\
+D_{\alpha}\left(b(\zeta)-a_{n}^{\prime}(\zeta)\right) \frac{\partial f}{\partial a_{\alpha}}\left(\pi_{a_{n}^{\prime}, u_{n}^{\prime}}(\zeta)\right) \\
\left.+\frac{1}{n(\beta, \gamma)} D_{\beta \gamma}^{2}\left(b(\zeta)-a_{n}^{\prime}(\zeta)\right) \frac{\partial f}{\partial a_{\beta \gamma}}\left(\pi_{a_{n}^{\prime}, u_{n}^{\prime}}(\zeta)\right)\right] d \zeta+\iota_{n} \geq 0, \quad \forall(b, w) \in A \times U .
\end{gathered}
$$

Clearly, it follows that $\left\{\left(a_{n}, u_{n}\right)\right\}$ and $\left.\left\{\left(a_{n}^{\prime}, u_{n}^{\prime}\right)\right)\right\}$ are two approximating sequences for (CVCP) which converge to $(\bar{a}, \bar{u})$ (by hypothesis, the problem (CVCP) is well posed). By computation, we obtain:

$$
\begin{gathered}
\left\|\left(a_{n}, u_{n}\right)-\left(a_{n}^{\prime}, u_{n}^{\prime}\right)\right\| \\
=\left\|\left(a_{n}, u_{n}\right)-(\bar{a}, \bar{u})+(\bar{a}, \bar{u})-\left(a_{n}^{\prime}, u_{n}^{\prime}\right)\right\| \\
\leq\left\|\left(a_{n}, u_{n}\right)-(\bar{a}, \bar{u})\right\|+\left\|(\bar{a}, \bar{u})-\left(a_{n}^{\prime}, u_{n}^{\prime}\right)\right\| \leq \iota,
\end{gathered}
$$

which contradicts (1), for some $\iota=r$. It follows that diam $\mathcal{S}(\sigma, \iota) \rightarrow 0$ as $(\sigma, \iota) \rightarrow(0,0)$.

Conversely, let $\left\{\left(a_{n}, u_{n}\right)\right\}$ be an approximating sequence for (CVCP). Therefore, there exists a sequence of positive real numbers $\iota_{n} \rightarrow 0$ as $n \rightarrow \infty$ such that the inequalities:

$$
\begin{gathered}
\lim _{n \rightarrow \infty} \sup \int_{\Omega} f\left(\pi_{a_{n}, u_{n}}(\zeta)\right) d \zeta \leq \inf _{(b, w) \in \Theta} \int_{\Omega} f\left(\pi_{b, w}(\zeta)\right) d \zeta \\
\int_{\Omega}\left[\left(b(\zeta)-a_{n}(\zeta)\right) \frac{\partial f}{\partial a}\left(\pi_{a_{n}, u_{n}}(\zeta)\right)+\left(w(\zeta)-u_{n}(\zeta)\right) \frac{\partial f}{\partial u}\left(\pi_{a_{n}, u_{n}}(\zeta)\right)\right. \\
+D_{\alpha}\left(b(\zeta)-a_{n}(\zeta)\right) \frac{\partial f}{\partial a_{\alpha}}\left(\pi_{a_{n}, u_{n}}(\zeta)\right) \\
\left.+\frac{1}{n(\beta, \gamma)} D_{\beta \gamma}^{2}\left(b(\zeta)-a_{n}(\zeta)\right) \frac{\partial f}{\partial a_{\beta \gamma}}\left(\pi_{a_{n}, u_{n}}(\zeta)\right)\right] d \zeta+\iota_{n} \geq 0, \quad \forall(b, w) \in A \times U
\end{gathered}
$$

hold, involving that $\left(a_{n}, u_{n}\right) \in \mathcal{S}\left(\sigma_{n}, l_{n}\right)$ (see $\sigma_{n} \rightarrow 0$ as $n \rightarrow \infty$, a sequence of positive real numbers). By considering diam $\mathcal{S}\left(\sigma_{n}, l_{n}\right) \rightarrow 0$ as $\left(\sigma_{n}, l_{n}\right) \rightarrow(0,0)$, we obtain that $\left\{\left(a_{n}, u_{n}\right)\right\}$ is a Cauchy sequence which converges to some $(\bar{a}, \bar{u}) \in A \times U$ as $A \times U$ is a closed set.

By using the monotonicity property of $\int_{\Omega} f\left(\pi_{a, u}(\zeta)\right) d \zeta$ on $A \times U$, for $(\bar{a}, \bar{u}),(b, w) \in$ $A \times U$, we have:

$$
\begin{aligned}
& \int_{\Omega}\left[(\bar{a}(\zeta)-b(\zeta))\left(\frac{\partial f}{\partial a}\left(\pi_{\bar{a}, \bar{u}}(\zeta)\right)-\frac{\partial f}{\partial a}\left(\pi_{b, w}(\zeta)\right)\right)\right. \\
& +(\bar{u}(\zeta)-w(\zeta))\left(\frac{\partial f}{\partial u}\left(\pi_{\bar{a}, \bar{u}}(\zeta)\right)-\frac{\partial f}{\partial a}\left(\pi_{b, w}(\zeta)\right)\right)
\end{aligned}
$$




$$
\begin{gathered}
+D_{\alpha}(\bar{a}(\zeta)-b(\zeta))\left(\frac{\partial f}{\partial a_{\alpha}}\left(\pi_{\bar{a}, \bar{u}}(\zeta)\right)-\frac{\partial f}{\partial a_{\alpha}}\left(\pi_{b, w}(\zeta)\right)\right) \\
\left.+\frac{1}{n(\beta, \gamma)} D_{\beta \gamma}^{2}(\bar{a}(\zeta)-b(\zeta))\left(\frac{\partial f}{\partial a_{\beta \gamma}}\left(\pi_{\bar{a}, \bar{u}}(\zeta)\right)-\frac{\partial f}{\partial a_{\beta \gamma}}\left(\pi_{b, w}(\zeta)\right)\right)\right] d \zeta \geq 0,
\end{gathered}
$$

or, equivalently:

$$
\begin{gathered}
\int_{\Omega}\left[(\bar{a}(\zeta)-b(\zeta)) \frac{\partial f}{\partial a}\left(\pi_{\bar{a}, \bar{u}}(\zeta)\right)+(\bar{u}(\zeta)-w(\zeta)) \frac{\partial f}{\partial u}\left(\pi_{\bar{a}, \bar{u}}(\zeta)\right)\right. \\
+D_{\alpha}(\bar{a}(\zeta)-b(\zeta)) \frac{\partial f}{\partial a_{\alpha}}\left(\pi_{\bar{a}, \bar{u}}(\zeta)\right) \\
\left.+\frac{1}{n(\beta, \gamma)} D_{\beta \gamma}^{2}(\bar{a}(\zeta)-b(\zeta)) \frac{\partial f}{\partial a_{\beta \gamma}}\left(\pi_{\bar{a}, \bar{u}}(\zeta)\right)\right] d \zeta \\
\geq \int_{\Omega}\left[(\bar{a}(\zeta)-b(\zeta)) \frac{\partial f}{\partial a}\left(\pi_{b, w}(\zeta)\right)+(\bar{u}(\zeta)-w(\zeta)) \frac{\partial f}{\partial u}\left(\pi_{b, w}(\zeta)\right)\right. \\
+D_{\alpha}(\bar{a}(\zeta)-b(\zeta)) \frac{\partial f}{\partial a_{\alpha}}\left(\pi_{b, w}(\zeta)\right) \\
\left.+\frac{1}{n(\beta, \gamma)} D_{\beta \gamma}^{2}(\bar{a}(\zeta)-b(\zeta)) \frac{\partial f}{\partial a_{\beta \gamma}}\left(\pi_{b, w}(\zeta)\right)\right] d \zeta .
\end{gathered}
$$

By considering the limit in inequality (3), we obtain:

$$
\begin{gathered}
\int_{\Omega}\left[(\bar{a}(\zeta)-b(\zeta)) \frac{\partial f}{\partial a}\left(\pi_{\bar{a}, \bar{u}}(\zeta)\right)+(\bar{u}(\zeta)-w(\zeta)) \frac{\partial f}{\partial u}\left(\pi_{\bar{a}, \bar{u}}(\zeta)\right)\right. \\
+D_{\alpha}(\bar{a}(\zeta)-b(\zeta)) \frac{\partial f}{\partial a_{\alpha}}\left(\pi_{\bar{a}, \bar{u}}(\zeta)\right) \\
\left.+\frac{1}{n(\beta, \gamma)} D_{\beta \gamma}^{2}(\bar{a}(\zeta)-b(\zeta)) \frac{\partial f}{\partial a_{\beta \gamma}}\left(\pi_{\bar{a}, \bar{u}}(\zeta)\right)\right] d \zeta \leq 0 .
\end{gathered}
$$

By using (4) and (5), it results that:

$$
\begin{gathered}
\int_{\Omega}\left[(b(\zeta)-\bar{a}(\zeta)) \frac{\partial f}{\partial a}\left(\pi_{b, w}(\zeta)\right)+(w(\zeta)-\bar{u}(\zeta)) \frac{\partial f}{\partial u}\left(\pi_{b, w}(\zeta)\right)\right. \\
+D_{\alpha}(b(\zeta)-\bar{a}(\zeta)) \frac{\partial f}{\partial a_{\alpha}}\left(\pi_{b, w}(\zeta)\right) \\
\left.+\frac{1}{n(\beta, \gamma)} D_{\beta \gamma}^{2}(b(\zeta)-\bar{a}(\zeta)) \frac{\partial f}{\partial a_{\beta \gamma}}\left(\pi_{b, w}(\zeta)\right)\right] d \zeta \geq 0 .
\end{gathered}
$$

Now, we use Lemma 1 to obtain:

$$
\begin{gathered}
\int_{\Omega}\left[(b(\zeta)-\bar{a}(\zeta)) \frac{\partial f}{\partial a}\left(\pi_{\bar{a}, \bar{u}}(\zeta)\right)+(w(\zeta)-\bar{u}(\zeta)) \frac{\partial f}{\partial u}\left(\pi_{\bar{a}, \bar{u}}(\zeta)\right)\right. \\
+D_{\alpha}(b(\zeta)-\bar{a}(\zeta)) \frac{\partial f}{\partial a_{\alpha}}\left(\pi_{\bar{a}, \bar{u}}(\zeta)\right) \\
\left.+\frac{1}{n(\beta, \gamma)} D_{\beta \gamma}^{2}(b(\zeta)-\bar{a}(\zeta)) \frac{\partial f}{\partial a_{\beta \gamma}}\left(\pi_{\bar{a}, \bar{u}}(\zeta)\right)\right] d \zeta \geq 0
\end{gathered}
$$

which implies that $(\bar{a}, \bar{u}) \in \Theta$. 
Since the functional $\int_{\Omega} f\left(\pi_{a, u}(\zeta)\right) d \zeta$ is lower semi-continuous, we conclude:

$$
\int_{\Omega} f\left(\pi_{\bar{a}, \bar{u}}(\zeta)\right) d \zeta \leq \lim _{n \rightarrow \infty} \inf \int_{\Omega} f\left(\pi_{a_{n}, u_{n}}(\zeta)\right) d \zeta \leq \lim _{n \rightarrow \infty} \sup \int_{\Omega} f\left(\pi_{a_{n}, u_{n}}(\zeta)\right) d \zeta .
$$

By (2), the previous inequality can be written as

$$
\int_{\Omega} f\left(\pi_{\bar{a}, \bar{u}}(\zeta)\right) d \zeta \leq \inf _{(b, w) \in \Theta} \int_{\Omega} f\left(\pi_{b, w}(\zeta)\right) d \zeta
$$

As a consequence, by (6) and (7), we obtain that $(\bar{a}, \bar{u})$ is the solution for (CVCP).

Let us prove that $(\bar{a}, \bar{u})$ is the single solution for (CVCP). Suppose that $\left(a_{1}, u_{1}\right) \neq$ $\left(a_{2}, u_{2}\right)$ are two solutions for (CVCP). Then:

$$
0<\left\|\left(a_{1}, u_{1}\right)-\left(a_{2}, u_{2}\right)\right\| \leq \operatorname{diam} \mathcal{S}(\sigma, \iota) \rightarrow 0 \text { as }(\sigma, l) \rightarrow(0,0),
$$

which is not possible. The proof is now complete.

The second main result of this paper is contained in the next theorem.

Theorem 2. Consider $F(a, u)=\int_{\Omega} f\left(\pi_{a, u}(\zeta)\right) d \zeta$ is lower semicontinuous, monotone and hemicontinuous on $A \times U$. The constrained optimization problem (CVCP) is well posed if and only if it admits a solution.

Proof. Consider that (CVCP) is well posed. In consequence, it has a solution $\left(a_{0}, u_{0}\right)$. Conversely, consider that $(\mathrm{CVCP})$ has a solution $\left(a_{0}, u_{0}\right)$, that is:

$$
\begin{gathered}
\int_{\Omega} f\left(\pi_{a_{0}, u_{0}}(\zeta)\right) d \zeta \leq \inf _{(b, w) \in \Theta} \int_{\Omega} f\left(\pi_{b, w}(\zeta)\right) d \zeta, \\
\int_{\Omega}\left[\left(b(\zeta)-a_{0}(\zeta)\right) \frac{\partial f}{\partial a}\left(\pi_{a_{0}, u_{0}}(\zeta)\right)+\left(w(\zeta)-u_{0}(\zeta)\right) \frac{\partial f}{\partial u}\left(\pi_{a_{0}, u_{0}}(\zeta)\right)\right. \\
+D_{\alpha}\left(b(\zeta)-a_{0}(\zeta)\right) \frac{\partial f}{\partial a_{\alpha}}\left(\pi_{a_{0}, u_{0}}(\zeta)\right) \\
\left.+\frac{1}{n(\beta, \gamma)} D_{\beta \gamma}^{2}\left(b(\zeta)-a_{0}(\zeta)\right) \frac{\partial f}{\partial a_{\beta \gamma}}\left(\pi_{a_{0}, u_{0}}(\zeta)\right)\right] d \zeta \geq 0, \quad \forall(b, w) \in A \times U,
\end{gathered}
$$

but (CVCP) is not well posed. Therefore, by Definition 6, there exists an approximating sequence $\left\{\left(a_{n}, u_{n}\right)\right\}$ of $(\mathrm{CVCP})$ (which does not converge to $\left.\left(a_{0}, u_{0}\right)\right)$, that is the following inequalities hold:

$$
\lim _{n \rightarrow \infty} \sup \int_{\Omega} f\left(\pi_{a_{n}, u_{n}}(\zeta)\right) d \zeta \leq \inf _{(b, w) \in \Theta} \int_{\Omega} f\left(\pi_{b, w}(\zeta)\right) d \zeta
$$

and:

$$
\begin{gathered}
\int_{\Omega}\left[\left(b(\zeta)-a_{n}(\zeta)\right) \frac{\partial f}{\partial a}\left(\pi_{a_{n}, u_{n}}(\zeta)\right)+\left(w(\zeta)-u_{n}(\zeta)\right) \frac{\partial f}{\partial u}\left(\pi_{a_{n}, u_{n}}(\zeta)\right)\right. \\
+D_{\alpha}\left(b(\zeta)-a_{n}(\zeta)\right) \frac{\partial f}{\partial a_{\alpha}}\left(\pi_{a_{n}, u_{n}}(\zeta)\right) \\
\left.+\frac{1}{n(\beta, \gamma)} D_{\beta \gamma}^{2}\left(b(\zeta)-a_{n}(\zeta)\right) \frac{\partial f}{\partial a_{\beta \gamma}}\left(\pi_{a_{n}, u_{n}}(\zeta)\right)\right] d \zeta+\iota_{n} \geq 0, \quad \forall(b, w) \in A \times U .
\end{gathered}
$$

Furthermore, to prove the boundedness of $\left\{\left(a_{n}, u_{n}\right)\right\}$, we proceed by contradiction. Suppose, in contrast to the result, $\left\{\left(a_{n}, u_{n}\right)\right\}$ is not bounded, that is, $\left\|\left(a_{n}, u_{n}\right)\right\| \rightarrow+\infty$ as $n \rightarrow+\infty$. Let us consider $\delta_{n}=\frac{1}{\left\|\left(a_{n}, u_{n}\right)-\left(a_{0}, u_{0}\right)\right\|}$ and $\left(\mathrm{a}_{n}, \mathrm{u}_{n}\right)=\left(a_{0}, u_{0}\right)+\delta_{n}\left[\left(a_{n}, u_{n}\right)-\right.$ 
$\left.\left(a_{0}, u_{0}\right)\right]$. We can see that $\left\{\left(a_{n}, u_{n}\right)\right\}$ is bounded in $A \times U$. If necessary, passing to a subsequence, we may consider that:

$$
\left(\mathrm{a}_{n}, \mathrm{u}_{n}\right) \rightarrow(\mathrm{a}, \mathrm{u}) \text { weakly in } A \times U \neq\left(a_{0}, u_{0}\right) .
$$

It is easy to check that $(\mathrm{a}, \mathrm{u}) \neq\left(a_{0}, u_{0}\right)$ thanks to $\left\|\delta_{n}\left[\left(a_{n}, u_{n}\right)-\left(a_{0}, u_{0}\right)\right]\right\|=1$, for all $n \in \mathbb{N}$. Since $\left(a_{0}, u_{0}\right)$ is a solution of (CVCP), the inequalities in (8) are satisfied. By Lemma 1 , we obtain:

$$
\begin{gathered}
\int_{\Omega} f\left(\pi_{a_{0}, u_{0}}(\zeta)\right) d \zeta \leq \inf _{(b, w) \in \Theta} \int_{\Omega} f\left(\pi_{b, w}(\zeta)\right) d \zeta \\
\int_{\Omega}\left[\left(b(\zeta)-a_{0}(\zeta)\right) \frac{\partial f}{\partial a}\left(\pi_{b, w}(\zeta)\right)+\left(w(\zeta)-u_{0}(\zeta)\right) \frac{\partial f}{\partial u}\left(\pi_{b, w}(\zeta)\right)\right. \\
+D_{\alpha}\left(b(\zeta)-a_{0}(\zeta)\right) \frac{\partial f}{\partial a_{\alpha}}\left(\pi_{b, w}(\zeta)\right) \\
\left.+\frac{1}{n(\beta, \gamma)} D_{\beta \gamma}^{2}\left(b(\zeta)-a_{0}(\zeta)\right) \frac{\partial f}{\partial a_{\beta \gamma}}\left(\pi_{b, w}(\zeta)\right)\right] d \zeta \geq 0, \quad \forall(b, w) \in A \times U .
\end{gathered}
$$

By using the monotonicity property of the multiple integral functional $\int_{\Omega} f\left(\pi_{a, u}(\zeta)\right) d \zeta$ on $A \times U$, for $\left(a_{n}, u_{n}\right),(b, w) \in A \times U$, we have:

$$
\begin{gathered}
\int_{\Omega}\left[\left(a_{n}(\zeta)-b(\zeta)\right)\left(\frac{\partial f}{\partial a}\left(\pi_{a_{n}, u_{n}}(\zeta)\right)-\frac{\partial f}{\partial a}\left(\pi_{b, w}(\zeta)\right)\right)\right. \\
+\left(u_{n}(\zeta)-w(\zeta)\right)\left(\frac{\partial f}{\partial u}\left(\pi_{a_{n}, u_{n}}(\zeta)\right)-\frac{\partial f}{\partial a}\left(\pi_{b, w}(\zeta)\right)\right) \\
+D_{\alpha}\left(a_{n}(\zeta)-b(\zeta)\right)\left(\frac{\partial f}{\partial a_{\alpha}}\left(\pi_{a_{n}, u_{n}}(\zeta)\right)-\frac{\partial f}{\partial a_{\alpha}}\left(\pi_{b, w}(\zeta)\right)\right) \\
\left.+\frac{1}{n(\beta, \gamma)} D_{\beta \gamma}^{2}\left(a_{n}(\zeta)-b(\zeta)\right)\left(\frac{\partial f}{\partial a_{\beta \gamma}}\left(\pi_{a_{n}, u_{n}}(\zeta)\right)-\frac{\partial f}{\partial a_{\beta \gamma}}\left(\pi_{b, w}(\zeta)\right)\right)\right] d \zeta \geq 0,
\end{gathered}
$$

or, equivalently:

$$
\begin{gathered}
\int_{\Omega}\left[\left(b(\zeta)-a_{n}(\zeta)\right) \frac{\partial f}{\partial a}\left(\pi_{a_{n}, u_{n}}(\zeta)\right)+\left(w(\zeta)-u_{n}(\zeta)\right) \frac{\partial f}{\partial u}\left(\pi_{a_{n}, u_{n}}(\zeta)\right)\right. \\
+D_{\alpha}\left(b(\zeta)-a_{n}(\zeta)\right) \frac{\partial f}{\partial a_{\alpha}}\left(\pi_{a_{n}, u_{n}}(\zeta)\right) \\
\left.+\frac{1}{n(\beta, \gamma)} D_{\beta \gamma}^{2}\left(b(\zeta)-a_{n}(\zeta)\right) \frac{\partial f}{\partial a_{\beta \gamma}}\left(\pi_{a_{n}, u_{n}}(\zeta)\right)\right] d \zeta \\
\leq \int_{\Omega}\left[\left(b(\zeta)-a_{n}(\zeta)\right) \frac{\partial f}{\partial a}\left(\pi_{b, w}(\zeta)\right)+\left(w(\zeta)-u_{n}(\zeta)\right) \frac{\partial f}{\partial u}\left(\pi_{b, w}(\zeta)\right)\right. \\
+D_{\alpha}\left(b(\zeta)-a_{n}(\zeta)\right) \frac{\partial f}{\partial a_{\alpha}}\left(\pi_{b, w}(\zeta)\right) \\
\left.+\frac{1}{n(\beta, \gamma)} D_{\beta \gamma}^{2}\left(b(\zeta)-a_{n}(\zeta)\right) \frac{\partial f}{\partial a_{\beta \gamma}}\left(\pi_{b, w}(\zeta)\right)\right] d \zeta
\end{gathered}
$$

Combining with (9) and (11), we have:

$$
\begin{gathered}
\int_{\Omega}\left[\left(b(\zeta)-a_{n}(\zeta)\right) \frac{\partial f}{\partial a}\left(\pi_{b, w}(\zeta)\right)+\left(w(\zeta)-u_{n}(\zeta)\right) \frac{\partial f}{\partial u}\left(\pi_{b, w}(\zeta)\right)\right. \\
+D_{\alpha}\left(b(\zeta)-a_{n}(\zeta)\right) \frac{\partial f}{\partial a_{\alpha}}\left(\pi_{b, w}(\zeta)\right)
\end{gathered}
$$




$$
\left.+\frac{1}{n(\beta, \gamma)} D_{\beta \gamma}^{2}\left(b(\zeta)-a_{n}(\zeta)\right) \frac{\partial f}{\partial a_{\beta \gamma}}\left(\pi_{b, w}(\zeta)\right)\right] d \zeta \geq-\iota_{n}, \quad \forall(b, w) \in A \times U
$$

Because of $\delta_{n} \rightarrow 0$ as $n \rightarrow \infty$ (by the assumption that $\left\{\left(a_{n}, u_{n}\right)\right\}$ is not bounded), so, we can take $n_{0} \in \mathbb{N}$ be large enough such that $\delta_{n}<1$, for all $n \geq n_{0}$. Then, by multiplying the previous inequality and (10) by $\delta_{n}>0$ and $1-\delta_{n}>0$, respectively, we obtain:

$$
\begin{gathered}
\int_{\Omega}\left[\left(b(\zeta)-a_{n}(\zeta)\right) \frac{\partial f}{\partial a}\left(\pi_{b, w}(\zeta)\right)+\left(w(\zeta)-u_{n}(\zeta)\right) \frac{\partial f}{\partial u}\left(\pi_{b, w}(\zeta)\right)\right. \\
+D_{\alpha}\left(b(\zeta)-a_{n}(\zeta)\right) \frac{\partial f}{\partial a_{\alpha}}\left(\pi_{b, w}(\zeta)\right) \\
\left.+\frac{1}{n(\beta, \gamma)} D_{\beta \gamma}^{2}\left(b(\zeta)-a_{n}(\zeta)\right) \frac{\partial f}{\partial a_{\beta \gamma}}\left(\pi_{b, w}(\zeta)\right)\right] d \zeta \geq-\iota_{n}, \quad \forall(b, w) \in A \times U, \forall n \geq n_{0} .
\end{gathered}
$$

Since $\left(\mathrm{a}_{n}, \mathrm{u}_{n}\right) \rightarrow(\mathrm{a}, \mathrm{u}) \neq\left(a_{0}, u_{0}\right)$ and $\left(\mathrm{a}_{n}, \mathrm{u}_{n}\right)=\left(a_{0}, u_{0}\right)+\left(\mathbf{a}_{n}, \mathbf{u}_{n}\right)\left[\left(a_{n}, u_{n}\right)-\left(a_{0}, u_{0}\right)\right]$, we have:

$$
\begin{gathered}
\int_{\Omega}\left[(b(\zeta)-\mathrm{a}(\zeta)) \frac{\partial f}{\partial a}\left(\pi_{b, w}(\zeta)\right)+(w(\zeta)-\mathrm{u}(\zeta)) \frac{\partial f}{\partial u}\left(\pi_{b, w}(\zeta)\right)\right. \\
+D_{\alpha}(b(\zeta)-\mathrm{a}(\zeta)) \frac{\partial f}{\partial a_{\alpha}}\left(\pi_{b, w}(\zeta)\right) \\
\left.+\frac{1}{n(\beta, \gamma)} D_{\beta \gamma}^{2}(b(\zeta)-\mathrm{a}(\zeta)) \frac{\partial f}{\partial a_{\beta \gamma}}\left(\pi_{b, w}(\zeta)\right)\right] d \zeta \\
=\lim _{n \rightarrow \infty} \int_{\Omega}\left[\left(b(\zeta)-\mathrm{a}_{n}(\zeta)\right) \frac{\partial f}{\partial a}\left(\pi_{b, w}(\zeta)\right)+\left(w(\zeta)-\mathrm{u}_{n}(\zeta)\right) \frac{\partial f}{\partial u}\left(\pi_{b, w}(\zeta)\right)\right. \\
+D_{\alpha}\left(b(\zeta)-\mathrm{a}_{n}(\zeta)\right) \frac{\partial f}{\partial a_{\alpha}}\left(\pi_{b, w}(\zeta)\right) \\
\left.+\frac{1}{n(\beta, \gamma)} D_{\beta \gamma}^{2}\left(b(\zeta)-\mathrm{a}_{n}(\zeta)\right) \frac{\partial f}{\partial a_{\beta \gamma}}\left(\pi_{b, w}(\zeta)\right)\right] d \zeta \\
\geq-\lim _{n \rightarrow \infty} \iota_{n}=0, \quad \forall(b, w) \in A \times U .
\end{gathered}
$$

By considering the lower semicontinuity of the considered functional, and taking into account Lemma 1, we have:

$$
\begin{gathered}
\int_{\Omega} f\left(\pi_{\mathrm{a}, \mathrm{u}}(\zeta)\right) d \zeta \leq \inf _{(b, w) \in \Theta} \int_{\Omega} f\left(\pi_{b, w}(\zeta)\right) d \zeta, \\
\int_{\Omega}\left[(b(\zeta)-\mathrm{a}(\zeta)) \frac{\partial f}{\partial a}\left(\pi_{\mathrm{a}, \mathrm{u}}(\zeta)\right)+(w(\zeta)-\mathrm{u}(\zeta)) \frac{\partial f}{\partial u}\left(\pi_{\mathrm{a}, \mathrm{u}}(\zeta)\right)\right. \\
+D_{\alpha}(b(\zeta)-\mathrm{a}(\zeta)) \frac{\partial f}{\partial a_{\alpha}}\left(\pi_{\mathrm{a}, \mathrm{u}}(\zeta)\right) \\
\left.+\frac{1}{n(\beta, \gamma)} D_{\beta \gamma}^{2}(b(\zeta)-\mathrm{a}(\zeta)) \frac{\partial f}{\partial a_{\beta \gamma}}\left(\pi_{\mathrm{a}, \mathrm{u}}(\zeta)\right)\right] d \zeta \geq 0, \quad \forall(b, w) \in A \times U .
\end{gathered}
$$

We obtain that $(\mathrm{a}, \mathrm{u})$ is a solution of (CVCP), which contradicts the uniqueness of $\left(a_{0}, u_{0}\right)$. In consequence, $\left\{\left(a_{n}, u_{n}\right)\right\}$ is a bounded sequence with a convergent subsequence $\left\{\left(a_{n_{k}}, u_{n_{k}}\right)\right\}$ which converges to $(\bar{a}, \bar{u}) \in A \times U$ as $k \rightarrow \infty$. Now, by Definition 1, for $\left(a_{n_{k}}, u_{n_{k}}\right),(b, w) \in$ $A \times U$, we have (see (11)):

$$
\begin{gathered}
\int_{\Omega}\left[\left(b(\zeta)-a_{n_{k}}(\zeta)\right) \frac{\partial f}{\partial a}\left(\pi_{a_{n_{k}}, u_{n_{k}}}(\zeta)\right)+\left(w(\zeta)-u_{n_{k}}(\zeta)\right) \frac{\partial f}{\partial u}\left(\pi_{a_{n_{k}}, u_{n_{k}}}(\zeta)\right)\right. \\
+D_{\alpha}\left(b(\zeta)-a_{n_{k}}(\zeta)\right) \frac{\partial f}{\partial a_{\alpha}}\left(\pi_{a_{n_{k_{k}}}, u_{n_{k}}}(\zeta)\right)
\end{gathered}
$$




$$
\begin{gathered}
\left.+\frac{1}{n(\beta, \gamma)} D_{\beta \gamma}^{2}\left(b(\zeta)-a_{n_{k}}(\zeta)\right) \frac{\partial f}{\partial a_{\beta \gamma}}\left(\pi_{a_{n_{k}}, u_{n_{k}}}(\zeta)\right)\right] d \zeta \\
\leq \int_{\Omega}\left[\left(b(\zeta)-a_{n_{k}}(\zeta)\right) \frac{\partial f}{\partial a}\left(\pi_{b, w}(\zeta)\right)+\left(w(\zeta)-u_{n_{k}}(\zeta)\right) \frac{\partial f}{\partial u}\left(\pi_{b, w}(\zeta)\right)\right. \\
+D_{\alpha}\left(b(\zeta)-a_{n_{k}}(\zeta)\right) \frac{\partial f}{\partial a_{\alpha}}\left(\pi_{b, w}(\zeta)\right) \\
\left.+\frac{1}{n(\beta, \gamma)} D_{\beta \gamma}^{2}\left(b(\zeta)-a_{n_{k}}(\zeta)\right) \frac{\partial f}{\partial a_{\beta \gamma}}\left(\pi_{b, w}(\zeta)\right)\right] d \zeta .
\end{gathered}
$$

Furthermore, on behalf of (9), we can write that:

$$
\begin{gathered}
\lim _{k \rightarrow \infty} \int_{\Omega}\left[\left(b(\zeta)-a_{n_{k}}(\zeta)\right) \frac{\partial f}{\partial a}\left(\pi_{a_{n_{k}}, u_{n_{k}}}(\zeta)\right)+\left(w(\zeta)-u_{n_{k}}(\zeta)\right) \frac{\partial f}{\partial u}\left(\pi_{a_{n_{k}}, u_{n_{k}}}(\zeta)\right)\right. \\
+D_{\alpha}\left(b(\zeta)-a_{n_{k}}(\zeta)\right) \frac{\partial f}{\partial a_{\alpha}}\left(\pi_{a_{n_{k}}, u_{n_{k}}}(\zeta)\right) \\
\left.+\frac{1}{n(\beta, \gamma)} D_{\beta \gamma}^{2}\left(b(\zeta)-a_{n_{k}}(\zeta)\right) \frac{\partial f}{\partial a_{\beta \gamma}}\left(\pi_{a_{n_{k}}, u_{n_{k}}}(\zeta)\right)\right] d \zeta \geq 0 .
\end{gathered}
$$

Combining (13) and (14), we have:

$$
\begin{gathered}
\lim _{k \rightarrow \infty} \int_{\Omega}\left[\left(b(\zeta)-a_{n_{k}}(\zeta)\right) \frac{\partial f}{\partial a}\left(\pi_{b, w}(\zeta)\right)+\left(w(\zeta)-u_{n_{k}}(\zeta)\right) \frac{\partial f}{\partial u}\left(\pi_{b, w}(\zeta)\right)\right. \\
+D_{\alpha}\left(b(\zeta)-a_{n_{k}}(\zeta)\right) \frac{\partial f}{\partial a_{\alpha}}\left(\pi_{b, w}(\zeta)\right) \\
\left.\quad+\frac{1}{n(\beta, \gamma)} D_{\beta \gamma}^{2}\left(b(\zeta)-a_{n_{k}}(\zeta)\right) \frac{\partial f}{\partial a_{\beta \gamma}}\left(\pi_{b, w}(\zeta)\right)\right] d \zeta \geq 0 \\
\Rightarrow \int_{\Omega}\left[(b(\zeta)-\bar{a}(\zeta)) \frac{\partial f}{\partial a}\left(\pi_{b, w}(\zeta)\right)+(w(\zeta)-\bar{u}(\zeta)) \frac{\partial f}{\partial u}\left(\pi_{b, w}(\zeta)\right)\right. \\
+D_{\alpha}(b(\zeta)-\bar{a}(\zeta)) \frac{\partial f}{\partial a_{\alpha}}\left(\pi_{b, w}(\zeta)\right) \\
\left.+\frac{1}{n(\beta, \gamma)} D_{\beta \gamma}^{2}(b(\zeta)-\bar{a}(\zeta)) \frac{\partial f}{\partial a_{\beta \gamma}}\left(\pi_{b, w}(\zeta)\right)\right] d \zeta \geq 0
\end{gathered}
$$

By considering the lower semicontinuity of the considered functional, in accordance with Lemma 1, we have:

$$
\begin{gathered}
\int_{\Omega} f\left(\pi_{\bar{a}, \bar{u}}(\zeta)\right) d \zeta \leq \inf _{(b, w) \in \Theta} \int_{\Omega} f\left(\pi_{b, w}(\zeta)\right) d \zeta \\
\int_{\Omega}\left[(b(\zeta)-\bar{a}(\zeta)) \frac{\partial f}{\partial a}\left(\pi_{\bar{a}, \bar{u}}(\zeta)\right)+(w(\zeta)-\bar{u}(\zeta)) \frac{\partial f}{\partial u}\left(\pi_{\bar{a}, \bar{u}}(\zeta)\right)\right. \\
+D_{\alpha}(b(\zeta)-\bar{a}(\zeta)) \frac{\partial f}{\partial a_{\alpha}}\left(\pi_{\bar{a}, \bar{u}}(\zeta)\right) \\
\left.+\frac{1}{n(\beta, \gamma)} D_{\beta \gamma}^{2}(b(\zeta)-\bar{a}(\zeta)) \frac{\partial f}{\partial a_{\beta \gamma}}\left(\pi_{\bar{a}, \bar{u}}(\zeta)\right)\right] d \zeta \geq 0
\end{gathered}
$$

implying that $(\bar{a}, \bar{u})$ is a solution for (CVCP). Therefore, $\left(a_{n_{k}}, u_{n_{k}}\right) \rightarrow(\bar{a}, \bar{u})$, that is, $\left(a_{n_{k}}, u_{n_{k}}\right) \rightarrow$ $\left(a_{0}, u_{0}\right)$, involving $\left(a_{n}, u_{n}\right) \rightarrow\left(a_{0}, u_{0}\right)$ and the proof is complete.

In the following illustrative example, we present an application of Theorems 1 and 2, as well. 
Example 2. Let us consider $n=k=1, m=2$, and $\Omega=[0,3]^{2}$. We define:

$$
f\left(\pi_{a, u}(\zeta)\right)=3 u^{2}(\zeta)+e^{a(\zeta)}-a(\zeta)
$$

and consider the following constrained variational control problem:

$$
(C V C P-1) \quad \text { Minimize }_{(a, u)} \int_{\Omega} f\left(\pi_{a, u}(\zeta)\right) d \zeta
$$

subject to

$$
\begin{gathered}
\int_{\Omega}\left[6(w(\zeta)-u(\zeta)) u(\zeta)+(b(\zeta)-a(\zeta))\left(e^{a(\zeta)}-1\right)\right] d \zeta \geq 0, \forall(b, w) \in A \times U \\
\left.(a, u)\right|_{\partial \Omega}=0 .
\end{gathered}
$$

We have $\mathcal{S}=\{(0,0)\}$. Moreover, we have that the functional $\int_{\Omega} f\left(\pi_{a, u}(\zeta)\right) d \zeta$ is monotone, hemicontinuous and lower semicontinuous on $A \times U=C^{4}(\Omega,[-10,10]) \times$ $C^{1}(\Omega,[-10,10])$. In consequence, all hypotheses in Theorem 2 hold, therefore the optimization problem (CVCP-1) is well posed. Furthermore, since $\mathcal{S}(\sigma, \iota)=\{(0,0)\}$, we obtain $\mathcal{S}(\sigma, \iota) \neq \varnothing$ and diam $\mathcal{S}(\sigma, \iota) \rightarrow 0$ as $(\sigma, \iota) \rightarrow(0,0)$. Consequently, by using Theorem 1, we obtain that the constrained optimization problem (CVCP-1) is well posed.

\section{Conclusions and Further Developments}

In this paper, we investigated the well posedness for a new class of constrained optimization problems governed by second-order partial derivatives. Concretely, by using the monotonicity, lower semicontinuity, pseudomonotonicity and hemicontinuity of multiple integral functional, we proved that the well posedness of the constrained optimization problem under study is characterized in terms of existence and uniqueness of solution. Furthermore, the theoretical developments have been accompanied by some examples.

Some further developments associated with the present paper are the following: to formulate the necessary and sufficient optimality conditions for the considered optimization problems, to establish some duality results, and to study the well posedness for similar classes of control problems by using fractional derivatives.

Funding: This research received no external funding.

Institutional Review Board Statement: Not applicable.

Informed Consent Statement: Not applicable.

Data Availability Statement: Not applicable.

Conflicts of Interest: The author declares no conflict of interest.

\section{References}

1. Tykhonov, A.N. On the stability of the functional optimization problems. USSR Comput. Math. Math. Phys. 1966, 6, 631-634. [CrossRef]

2. Hu, R.; Fang, Y.P. Levitin-Polyak well-posedness by perturbations of inverse variational inequalities. Optim. Lett. 2013, 7, 343-359. [CrossRef]

3. Jiang, B.; Zhang, J.; Huang, X.X. Levitin-Polyak well-posedness of generalized quasivariational inequalities with functional constraints. Nonlinear Anal. TMA 2009, 70, 1492-1503. [CrossRef]

4. Lalitha, C.S.; Bhatia, G. Levitin-Polyak well-posedness for parametric quasivariational inequality problem of the Minty type. Positivity 2012, 16, 527-541. [CrossRef]

5. Levitin, E.S.; Polyak, B.T. Convergence of minimizing sequences in conditional extremum problems. Sov. Math. Dokl. 1996, 7, 764-767.

6. Čoban, M.M.; Kenderov, P.S.; Revalski, J.P. Generic well-posedness of optimization problems in topological spaces. Mathematika 1989, 36, 301-324. [CrossRef]

7. Dontchev, A.L.; Zolezzi, T. Well-Posed Optimization Problems; Springer: Berlin, Germany, 1993. 
8. Furi, M.; Vignoli, A. A characterization of well-posed minimum problems in a complete metric space. J. Optim. Theory Appl. 1970, 5, 452-461. [CrossRef]

9. Huang, X.X. Extended and strongly extended well-posedness of set-valued optimization problems. Math. Methods Oper. Res. 2001, 53, 101-116. [CrossRef]

10. Huang, X.X.; Yang, X.Q. Generalized Levitin-Polyak well-posedness in constrained optimization. SIAM J. Optim. 2006, 17, 243-258. [CrossRef]

11. Lignola, M.B.; Morgan, J. Well-posedness for optimization problems with constraints defined by variational inequalities having a unique solution. J. Glob. Optim. 2000, 16, 57-67. [CrossRef]

12. Lin, L.J.; Chuang, C.S. Well-posedness in the generalized sense for variational inclusion and disclusion problems and wellposedness for optimization problems with constraint. Nonlinear Anal. 2009, 70, 3609-3617. [CrossRef]

13. Lucchetti, R. Convexity and Well-Posed Problems; Springer: New York, NY, USA, 2006.

14. Zolezzi, T. Extended well-posedness of optimization problems. J. Optim. Theory Appl. 1996, 91, 257-266. [CrossRef]

15. Lignola, M.B. Well-posedness and L-well-posedness for quasivariational inequalities. J. Optim. Theory Appl. 2006, 128, 119-138. [CrossRef]

16. Lignola, M.B.; Morgan, J. Approximate Solutions and $\alpha$-Well-Posedness for Variational Inequalities and Nash Equilibria, Decision and Control in Management Science; Zaccour, G., Ed.; Kluwer Academic Publishers: Dordrecht, The Netherlands, 2002 ; pp. 367-378.

17. Virmani, G.; Srivastava, M. On Levitin-Polyak $\alpha$-well-posedness of perturbed variational-hemivariational inequality. Optimization 2015, 64, 1153-1172. [CrossRef]

18. Ceng, L.C.; Yao, J.C. Well-posedness of generalized mixed variational inequalities, inclusion problems and fixed-point problems. Nonlinear Anal. 2008, 69, 4585-4603. [CrossRef]

19. Ceng, L.C.; Hadjisavvas, N.; Schaible, S.; Yao, J.C. Well-posedness for mixed quasivariational-like inequalities. J. Optim. Theory Appl. 2008, 139, 109-125. [CrossRef]

20. Fang, Y.P.; Hu, R. Estimates of approximate solutions and well-posedness for variational inequalities. Math. Meth. Oper. Res. 2007, 65, 281-291. [CrossRef]

21. Hammad, H.A.; Aydi, H.; la Sen, M.D. New contributions for tripled fixed point methodologies via a generalized variational principle with applications. Alex. Eng. J. 2021, in press. [CrossRef]

22. Lalitha, C.S.; Bhatia, G. Well-posedness for parametric quasivariational inequality problems and for optimization problems with quasivariational inequality constraints. Optimization 2010, 59, 997-1011. [CrossRef]

23. Xiao, Y.B.; Yang, X.M.; Huang, N.J. Some equivalence results for well-posedness of hemivariational inequalities. J. Glob. Optim. 2015, 61, 789-802. [CrossRef]

24. Heemels, P.M.H.; Camlibel, M.K.C.; Schaft, A.J.V.; Schumacher, J.M. Well-posedness of the complementarity class of hybrid systems. In Proceedings of the 15th IFAC Triennial World Congress, Barcelona, Spain, 21-26 July 2002.

25. Chen, J.W.; Wang, Z.; Cho, Y.J. Levitin-Polyak well-posedness by perturbations for systems of set-valued vector quasi-equilibrium problems. Math. Meth. Oper. Res. 2013, 77, 33-64. [CrossRef]

26. Fang, Y.P.; Hu, R.; Huang, N.J. Well-posedness for equilibrium problems and for optimization problems with equilibrium constraints. Comput. Math. Appl. 2008, 55, 89-100. [CrossRef]

27. Lignola, M.B.; Morgan, J. $\alpha$-Well-posedness for Nash equilibria and for optimization problems with Nash equilibrium constraints. J. Glob. Optim. 2006, 36, 439-459. [CrossRef]

28. Agarwal, P.; Filali, D.; Akram, M.; Dilshad, M. Convergence Analysis of a Three-Step Iterative Algorithm for Generalized Set-Valued Mixed-Ordered Variational Inclusion Problem. Symmetry 2021, 13, 444. [CrossRef]

29. Jayswal, A.; Jha, S. Well-posedness for generalized mixed vector variational-like inequality problems in Banach space. Math Commun. 2017, 22, 287-302.

30. Treanţă, S. A necessary and sufficient condition of optimality for a class of multidimensional control problems. Optim. Control Appl. Meth. 2020, 41, 2137-2148. [CrossRef]

31. Treanţă, S.; Arana-Jiménez, M.; Antczak, T. A necessary and sufficient condition on the equivalence between local and global optimal solutions in variational control problems. Nonlinear Anal. 2020, 191, 111640. [CrossRef]

32. Treanţă, S. On a modified optimal control problem with first-order PDE constraints and the associated saddle-point optimality criterion. Eur. J. Control 2020, 51, 1-9. [CrossRef]

33. Treanţă, S. Some results on $(\rho, b, d)$-variational inequalities. J. Math. Ineq. 2020, 14, 805-818. [CrossRef]

34. Treanţă, S. On weak sharp solutions in $(\rho, b, d)$-variational inequalities. J. Ineq. Appl. 2020, 2020, 54. [CrossRef]

35. Treanţă, S.; Singh, S. Weak sharp solutions associated with a multidimensional variational-type inequality. Positivity 2021, 25, 329-351. [CrossRef]

36. Treanţă, S.; Das, K. On robust saddle-point criterion in optimization problems with curvilinear integral functionals. Mathematics 2021, 9, 1790. [CrossRef]

37. Treanţă, S. On well-posed isoperimetric-type constrained variational control problems. J. Differ. Eq. 2021, 298, 480-499. [CrossRef]

38. Treanţă, S. Second-order PDE constrained controlled optimization problems with application in mechanics. Mathematics 2021, 9, 1472. [CrossRef]

39. Treanţă, S. On a class of second-order PDE\&PDI constrained robust modified optimization problems. Mathematics 2021, 9, 1473.

40. Treanţă, S. On a class of isoperimetric constrained controlled optimization problems. Axioms 2021, 10, 112. [CrossRef] 
41. Saunders, D.J. The Geometry of Jet Bundles, London Math. Soc. Lecture; Notes Series, 142; Cambridge University Press: Cambridge, UK, 1989.

42. Usman, F.; Khan, S.A. A generalized mixed vector variational-like inequality problem. Nonlinear Anal. 2009, 71, 5354-5362. [CrossRef] 